\title{
Study of Tumour Global DNA Methylation and mTOR Pathway Genes Promoters Methylation in Maternally Leucine-Supplemented Tumour-Bearing Adult Rats.
}

\section{Beatriz Schincariol Manhe*, Sarah Christine Pereira de Oliveira, Natália Angelo da Silva Miyaguti, Murilo Vieira Geraldo, Maria Cristina Cintra Gomes-Marcondes}

\begin{abstract}
Cancer-cachexia is a multifactorial syndrome characterised by negative protein balance, associated with alterations in Akt/mTOR (protein synthesis) and Ubiquitin-proteasome (protein degradation) pathways. Maternal supplementation with the amino acid leucine during pregnancy and lactation can ameliorate muscle loss in adult offspring tumour-bearing rats. The mTOR pathway proteins expression is altered in this situation, and these modifications may be associated with epigenetic modifications. Therefore, in this study, we aimed to analyse the methylation in CpG islands of Pik3r6, mTOR and RPS6KB1 promoters in muscle and the global DNA methylation in tumour tissue in order to investigate the possible epigenetic modifications provided by the maternal diet in the offspring.
\end{abstract}

Key words: Cancer-cachexia, leucine, epigenetics.

\section{Introduction}

Cachexia is a multifactorial metabolic syndrome, characterised by progressive wasting of both skeletal muscle and adipose tissue, which can affect nearly $85 \%$ of advanced cancer patients. In tumour-bearing rats, this muscle mass loss is due to a negative protein balance, usually associated to a decreased expression of Akt/mTOR pathway and increased expression of Ubiquitin-proteasome pathway, responsible for protein synthesis and degradation, respectively.

Our recent publication has first demonstrated that maternal supplementation with the branched-chain amino acid leucine can be used in a preventive approach against the waste of cancer cachexia. During pregnancy and lactation, the maternal diet has an important modulatory role in the offspring metabolic programming that could imply in permanent changes until adulthood. We observed the maintenance of muscle PI3K, mTOR and P70S6K proteins expressions in adult offspring tumour-bearing rats from dams supplemented with a leucine-rich diet when compared to the tumour-bearing group, in which these expressions were extensively altered.

These protein contents preservation may be due to a differential gene expression modulation induced by epigenetic modifications, such as DNA methylation in CpG islands, which leads to gene downregulation. Moreover, tumour tissue genes are expected to be hypomethylated. Therefore, in this study, we analysed the global DNA methylation in tumour tissue to investigate the influence of maternal supplemented diet. Also, we are evaluating the muscle methylation profile in the $\mathrm{CpG}$ islands of gene promoter regions of the genes Pik3r6, mTOR and RPS6KB1.

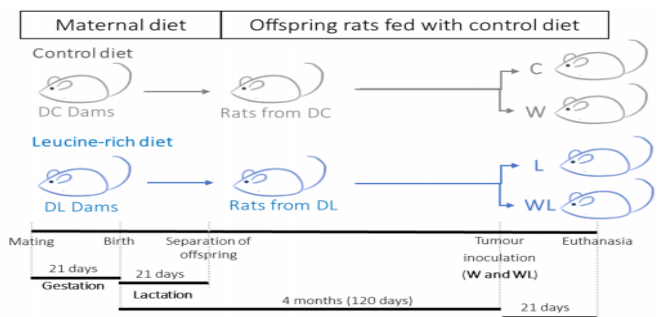

\section{Results and Discussion}

The global DNA methylation was analysed on tumour samples following the instructions of the 5-mC DNA ELISA Kit Zymo Research kit, based on a standard curve generated with known concentrations of 5-mC controls provided $(100 \%, 75 \%, 50 \%, 25 \%, 10 \%, 5 \%$ and $0 \%$ of $5-$ $\mathrm{mC}$ ). Using the absorbance values of the standard curve, it was possible to quantify the $5-\mathrm{mC}$ percentages of the samples in both groups and compared statistically using a Student's t-test (significant when $\mathrm{p}<0.05$ ).

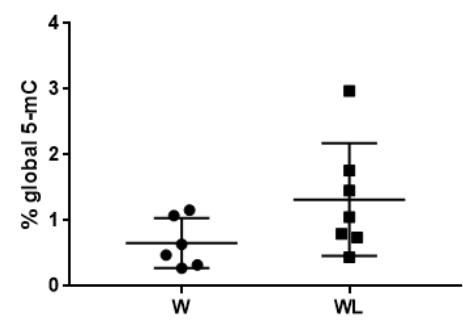

Image 2. Tumour global DNA methylation of W and WL groups.

As expected, the tumour genomic DNA showed a hypomethylated pattern. However, no differences were induced by the maternal leucine-rich diet $(\mathrm{W} \times \mathrm{WL}, \mathrm{p}=$ 0.1375).

\section{Conclusions}

The maternal leucine-rich diet was not able to modify the global DNA methylation in tumour tissue. In ongoing assays, the specific methylation profile of $\mathrm{CpG}$ islands in gene promoter regions of the genes Pik3r6, mTOR and RPS6KB1 will be analysed.

\section{Acknowledgement}

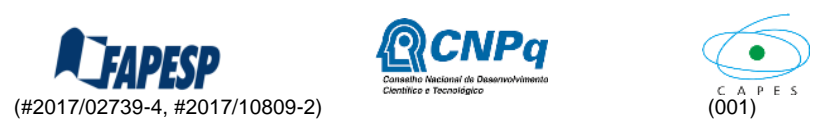

1. Argilés, J.M.; Stemmler, B.; López-Soriano, F.J.; Busquets, S. Inter-tissue communication in cancer cachexia. Nat. Rev. Endocrinol. 2018

2. Miyaguti, N.A.S.; Oliveira, S.C.P.; Gomes-Marcondes, M.C.C. Materna Leucine-Rich Diet Minimises Muscle Mass Loss in Tumour-bearing Adult Rat Offspring by Improving the Balance of Muscle Protein Synthesis and Degradation. Biomolecules 2019, 9, 229.

Image 1. Experimental procedure (Biomolecules, Miyaguti et. al, 2019). 